\title{
ENFOQUE DE LA INGENIERÍA DE REQUERIMIENTOS EN EL PERFECCIONAMIENTO CURRICULAR DE LA ESCUELA DE INGENIERÍA DE SISTEMAS DE LA UNIVERSIDAD PRIVADA DE TACNA 2008 - 2009
}

\author{
Autora: Mariella Rosario Ibarra Montecinos' \\ Universidad Privada de Tacna \\ E-mail: mariellaibarra@yahoo.es
}

\section{RESUMEN}

La investigación tuvo como objetivo aplicar la Ingeniería de Requerimientos al Perfeccionamiento Curricular como una solución que mejora la eficacia en los egresados de la Escuela de Ingeniería de Sistemas de la Universidad Privada de Tacna.

El método de investigación es explicativo porque se centra en responder a la pregunta ¿Por qué es asi la realidad? o ¿Cuáles son las causas?.

El resultado permitió determinar los indicadores y el nivel de dominio de las competencias alcanzadas por los egresados. Dichos niveles van transitando desde Novato hacia Experto; para la validación del logro de las competencias en cada ciclo de cambio se aplicaron encuestas a los egresados, lo que permitió la proyección de eficacia de cada una de las competencias para los siguientes ciclos de cambio.

Como conclusión, se tiene que la Ingeniería de requerimientos permitió establecer una proyección de logro Óptimo y Aceptable de las competencias generales, incorporar la proyección del logro de las competencias al Perfeccionamiento Curricular e identificar las brechas (de las competencias que no se cumplen con los niveles de logro esperados).

\section{PALABRAS CLAVE:}

Brecha de competencia: Es la brecha existente entre competencia impartida en la universidad y la competencia lograda en el centro laboral por un egresado, A menor brecha la competencia es eficaz a mayor brecha la competencia es ineficaz.

Ciclo de Cambio: Periodo en el cual se evalúan los indicadores de las competencias.

Perfeccionamiento Curricular: Es un proceso permanente, que no termina con el diseño de los nuevos planes de estudio, ya que la transformación que se busca es metodológica.

Ingeniería de Requerimientos: Es un proceso que comprende las tareas relacionadas con la determinación de los requerimientos a satisfacer para un nuevo o modificado sistema, al aplicarlo en esta investigación asegura que ningún requerimiento determinante en la formación de los egresados sea obviado.

Indicador de Competencia: Los indicadores deben permitir objetivar el nivel de dominio alcanzado por el egresado y los niveles de la competencia que se trate, dichos niveles se han de establecer de forma convencional, pero de una forma $u$ otra van transitando de niveles de Novato hacia el logro de niveles de Experto.

\begin{abstract}
The objective of this research work was to apply requirement engineering to the remodeling of curriculum as a solution to improve efficiency in graduates of school of systems engineering in the Private University of Tacna.

The investigation method is explanatory since it tries to answer the question: Why is the reality this way?, which are its causes?

Results obtained allowed us to determine the indicators and the level of competencies achieved by graduates. Such levels go from novice to expert. In order to validate competencies polls were applied to graduates in every level of change. This technique allowed to Project efficacy for next levels of change.

In conclusion, we see that requirements engineering allows to establish a goal of optimum performance and acceptable level in general competencies as well as to incorporate competencies in curricular perfectioning and also identify gaps (for competencies that are not fulfilled in the expected levels of achievement).
\end{abstract}

\section{KEYWORDS:}

Gaps competition, change cycles, Curriculum Development, Requirements Engineering, and Competition Indicator. 


\section{INTRODUCCIÓN}

Existen egresados de la Escuela de Ingenieria de Sistemas de la Universidad Privada de Tacna que presentan dificultades al insertarse en el mercado laboral, debido a que al egresar lo hicieron con un Plan Estudios resultado de la aplicación de un diseño curricular ineficaz en el que no se consideró una metodología específica, por lo que se requiere aplicar un perfeccionamiento curricular.

Cada vez que surge una innovación techológica o un cambio en el entorno, trae como consecuencia, la actualización de la secuencia y contenido de los componentes del currículo. Si la actualización no se ejecuta, afectará negativamente al producto, en este caso, los egresados, que pueden ser rechazados.

De acuerdo a la tendencia estadística se puede apreciar que algunos egresados están desempeñando puestos ocupacionales que no están relacionados con la especialidad ni insertados adecuadamente en el mercado laboral.

Lo que se pretende con esta investigación, es alcanzar un Perfeccionamiento Curricular eficaz, de tal manera que permita formar a los egresados de la Escuela de Ingeniería de Sistemas de la Universidad Privada de Tacna. Un curriculo actualizado, les permitirá competir en el mercado actual que es altamente competitivo; $y$, que sean aceptados por las empresas. La aplicación de la Ingenieria de Requerimientos asegura que ningún requisito determinante en la formación de los egresados no sea considerado, y obtengamos como resultado un egresado de Ingenieria de Sistemas con una formación óptima.

Los objetivos son:

- Aplicar la Ingeniería de Requerimientos al Perfeccionamiento Curricular para mejorar la eficacia en los egresados de Escuela de Ingenieria de Sistemas de la Universidad Privada de Tacna.

- Determinar los Indicadores de Logro de Competencias y su evaluación cuyos resultados sean aprovechables por el Perfeccionamiento Curricular de la Escuela de Ingeniería de Sistemas de la Universidad Privada de Tacna.

En la Hipótesis se consideró que la Ingeniería de Requerimientos permitirá la operatividad del Perfeccionamiento Curricular, y por tanto se logrará eficacia en la formación de los egresados de Ingeniería de Sistemas de la Universidad Privada de Tacna.

Esta investigación se hace necesaria porque busca mejorar los lineamientos metodológicos en la elaboración del currículo que actualmente existe en la Escuela de Ingeniería de Sistemas de la Universidad Privada de Tacna; puesto que muchas veces se desarrollan cambios curriculares sin un proceso metodológico adecuado, ni con una base científica sólida en función a las exigencias de la realidad social. La presente investigación aplicó un modelo curricular que satisface los requerimientos de dinámica del entorno y de los avances cientificostecnológicos.

La investigación plantea teorías de Ingenieria de Requerimientos y Perfeccionamiento Curricular que se contrastó con diferentes conceptos del diseño curricular y aspectos relacionados a los planes de mejora continua, que permitieron la retroalimentación curricular así como la identificación de los gaps (brechas) de las competencias que no se alcanzaron.

De acuerdo con los objetivos de la investigación, su resultado permite encontrar soluciones concretas a problemas de cambios en la dinámica del entorno que no habian sido incorporados en el Proceso de Perfeccionamiento Curricular, y que son capturados como requerimientos.

\section{MÉTODOSY MATERIALES}

\section{Metodología \\ Tipo de Investigación: Aplicativa}

Nivel de Investigación: Además de Descriptiva la Hipótesis es Explicativa.

Método de la Investigación: Método Explicativo, porque se centra en responder a la pregunta ¿Por qué es así la realidad? ¿ ¿Cuáles son las causas?.

\section{Cobertura del Estudio}

Universo: Escuela de Ingenieria de Sistemas de la Universidad Privada de Tacna.

Muestra: Egresados de los últimos 2 años de la Escuela de Ingeniería de Sistemas de la Universidad Privada de Tacna.

Técnicas de Recolección de datos: Encuesta

Instrumentos: Cuestionarios aplicados a Egresados.

\section{Modelos de Sistemas}

Se pueden desarrollar modelos de sistemas durante el proceso de ingeniería de requerimientos. Los requerimientos del usuario deberían redactarse en lenguaje natural debido a que tienen que ser comprendidos por personas que no son técnicos expertos. Sin embargo, 
pueden expresarse requerimientos del sistema más detallados de forma más técnica. Una técnica ampliamente usada es documentar la especificación del sistema como un conjunto de modelos del sistema. Estos modelos son representaciones gráficas que describen los procesos del negocio, el problema a resolver y el sistema que tiene que ser desarrollado. ${ }^{1}$

\section{Perfeccionamiento Curricular}

El diseño curricular se inicia con la elaboración del perfil de egreso. Éste será el resultado de valorar las competencias profesionales para un desempeño eficiente en su futura vida laboral dentro de su esfera de acción. ${ }^{2}$

\section{APLICACIÓN DEL PERFECCIONAMIENTO CURRICULAR}

En la Escuela de Ingeniería de Sistemas se ha asumido el Perfeccionamiento Curricular como un ejercicio académico que permite la actualización permanente de la propuesta formativa de la carrera.

\section{ACTIVIDAD 1:}

\section{ESTUDIO DE VIABILIDAD}

Comprende la evaluación y recopilación de la información; se consulta con las fuentes de información para descubrir las respuestas a estas preguntas:

¿Cómo se las arreglaría la Escuela si no se implementara el Sistema de Perfeccionamiento Curricular?

Se corre el riesgo de que el currículo no tenga Pertinencia e Impacto Social; por lo tanto, habría un incremento de la insatisfacción de los empleadores y los egresados con la calidad de la formación de los profesionales.

¿Cuál es la contribución que hará el Sistema de Perfeccionamiento Curricular a los objetivos y requerimientos de la Escuela?

Contribuir a la sostenibilidad de la carrera incrementando el número de ingresantes a la Escuela. Diferenciar a la Escuela de la competencia, al contar con un Plan de Estudios Competitivo.

ACTIVIDAD 2:

O B T E N C I Ó N Y A N ÁL IS I S D E REQUERIMIENTOS

Una vez recopilados los requisitos, estos se agrupan por categorias y se organizan en subconjuntos.

\section{ACTIVIDAD 3: ESPECIFICACIÓN DE REQUERIMIENTOS DEL SISTEMA}

\begin{tabular}{l|l|l}
\hline $\begin{array}{l}\text { Cojdigo } \\
\text { Requerimienb }\end{array}$ & $\begin{array}{l}\text { Tipo de } \\
\text { Requenmiento }\end{array}$ & \\
\hline Rf 1 & Funcional & Problema de la Profesión \\
\hline Rf 2 & Funcional & Análisis del Macro Contexto - Micro Conlexto \\
\hline Rf 3 & Funcional & Objetivos Educacionales Profesionales \\
\hline Rf 4 & Funcional & Macrodiseño Cumcular \\
\hline Rf 4.1 & Funcional & Objeto de Trabajo \\
\hline Rf 4.2 & Funcional & Objeto de la Profesión \\
\hline Rf 4.3 & Funcional & Perfiles Profesionales o Campos de Acción \\
\hline Rf 4.4 & Funcional & Perfiles Ocupacionales o Esferas de Actuación \\
\hline Rf 45 & Funcional & Areas de Conocimiento \\
\hline Rf5 & Funcional & Microdiseño Curricular \\
\hline Rf 5.1 & Funcional & Competencias de la Profesión \\
\hline Rf 5.2 & Funcional & Compelencias Terminales de los cursos \\
\hline Rf 6 & Funcional & Matriz Cumicular \\
\hline Rf 7 & Funcional & Cursos y otras actividades de aprendizaje \\
\hline Rf8 & Funcional & Ejecución de Evaluación de las Competencias \\
\hline Rf 9 & Funcional & Gestión de Requenimientos \\
\hline Rf 10 & Funcional & Realiza cambios \\
\hline Rf 11 & Funcional & Evalúa cambios \\
\hline Rnf 1 & No Funcional & Componente Investigativo \\
\hline Rnf 2 & No Funcional & Componente Laboral \\
\hline & &
\end{tabular}

\section{Rf 1: Problema de la Profesión}

Es el ¿dónde? de la profesión, y precisa los puestos de trabajo en que una vez graduados se ubicarán los profesionales.

\section{Rf 2: Análisis del Macro-Micro Contexto}

\section{Rf 2.1: Análisis del Macro Contexto}

Fuerzas del medio ambiente que a nivel macro influyen o afectan el comportamiento de la carrera.

\begin{tabular}{|l|l|l|}
\hline \multicolumn{1}{|c|}{ Fuerza } & $\begin{array}{l}\text { Grado de } \\
\text { Impacto }\end{array}$ & Naturaleza de Influencia \\
\hline $\begin{array}{l}\text { Universidades } \\
\text { Privadas de la } \\
\text { Región }\end{array}$ & Medio & $\begin{array}{l}\text { Competencia que puede } \\
\text { influir en la disminución } \\
\text { de los posibles } \\
\text { postulantes }\end{array}$ \\
\hline $\begin{array}{l}\text { Institutos } \\
\text { Tecnológicos }\end{array}$ & Medio & $\begin{array}{l}\text { Ofertan carreras técnicas } \\
\text { relacionada a la a a a } \\
\text { Ingeniería de Sistemas a } \\
\text { precios más económicos }\end{array}$ \\
\hline
\end{tabular}

\section{Rf 2.2: Análisis del micro contexto}

$\begin{array}{ll}\text { - } & \text { Pertinencia } \\ \text { - } & \text { Soporte Académico } \\ \text { - } & \text { Infraestructura } \\ & \text { Vida Profesional }\end{array}$


Rf 3: Objetivos Educacionales Profesionales

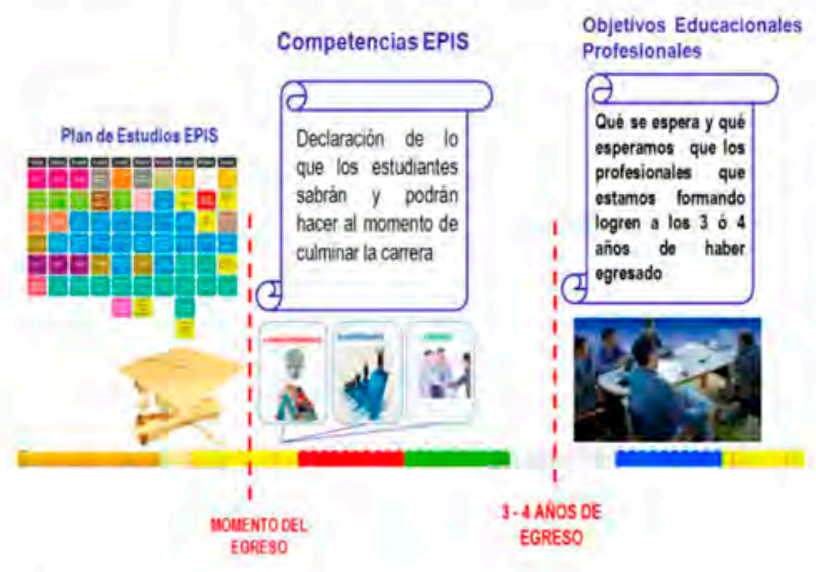

A través de los OEP la Escuela está respondiendo a las necesidades de los constituyentes.

\section{Objetivos Educacionales}

\section{Profesionales Competentes}

Ser efectivos en el diseño de soluciones tecnológicas y la aplicación práctica de principios de ciencia e ingeniería.

Desarrollo de Soluciones Integrales

Gestiona, desarrolla y aplica Proyectos de Sistemas y Tecnología de Información que agregan valor a las organizaciones y les permite competir en un entorno globalizado.

\section{Creatividad, colaboración y comunicación}

Poseen habilidades de solución analítica de problemas, y serán miembros de equipos multidisciplinarios ejerciendo diferentes roles y capaces de comunicar efectivamente sus ideas.

\section{Aprendizaje permanente}

Los graduados aprenderán y aplicarán nuevas y cambiantes tecnologías y proseguirán estudios de especialización.

\section{Rf 4: Macrodiseño Curricular \\ Rf 4.1: Objeto de Trabajo}

Es aquello que recibe la acción, lo que es transformado. En este caso: Sistemas de Información.

\section{Rf 4.2: Objeto de la Profesión \\ Integración de Sistemas}

\section{Rf 4.3: Perfiles Profesionales o Campos de Acción}

Se refiere a los conocimientos, habilidades y valores que tienen que asimilar, desarrollar y aplicar los estudiantes durante el proceso de formación en un perfil del saber de una carrera.
Rf 4.4: Perfiles Ocupacionales o Esferas de Actuación Es el ¿dónde? de la profesión, y precisa los puestos de trabajo en que, una vez graduados, se ubicarán los profesionales.

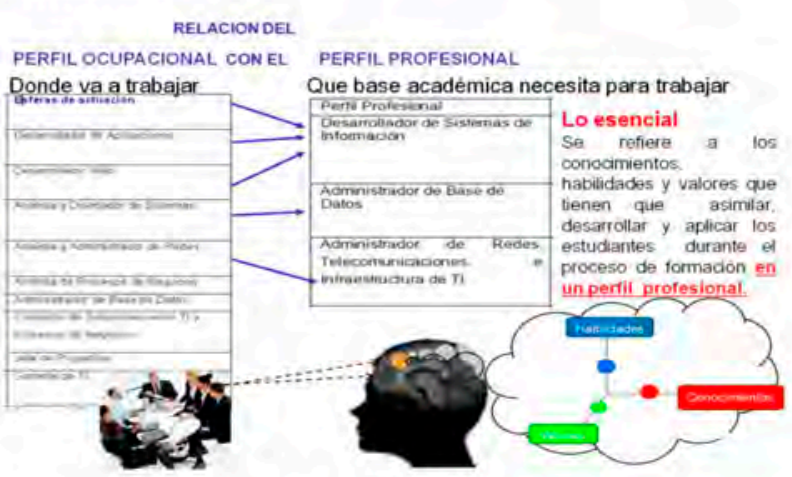

Rf4.5: Áreas de Conocimiento:

El área académica es un subsistema en el que los cursos se organizan en forma de sistema; ordenados lógica y pedagógicamente.

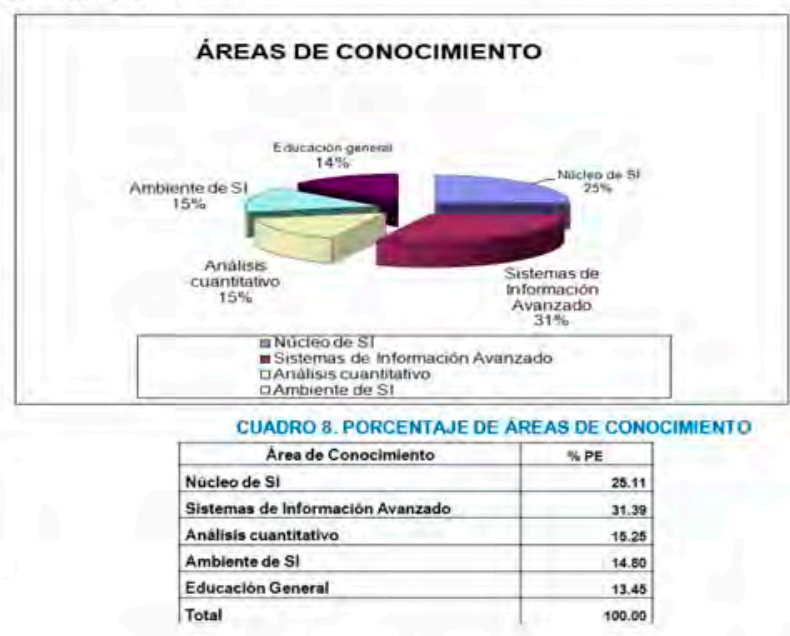

\section{Rf 5: Microdiseño curricular}

Rf 5.1: Modos de Actuación (Competencias de la Profesión)

\section{COMPETENCIA POR PERFIL PROFESIONAL}

Perfil Profesional

Desarrollador de Sistemas de Información

\section{Competencia}

Analiza, diseña e implementa sistemas de información, aplicando metodologías y tecnologías de información, para proponer soluciones informáticas que permitan el desarrollo de las organizaciones a través de la creación de valor y la mejora continua de sus operaciones y toma de decisiones. 


\section{COMPETENCIAS DE LA PROFESIÓN}

\begin{tabular}{|c|c|}
\hline $\begin{array}{l}\text { Competencia } \\
\text { relacionada a: }\end{array}$ & $\begin{array}{l}\text { Competencias de la } \\
\text { Profesión }\end{array}$ \\
\hline $\begin{array}{l}\text { Perfil Profesional } \\
\text { Desarrollador de } \\
\text { Sistemas de } \\
\text { Información }\end{array}$ & $\begin{array}{l}\text { Modela, construye y gestiona la } \\
\text { arquitectura de datos de la } \\
\text { organización, utilizando programas } \\
\text { de administración de base de datos } \\
\text { que permitan administrar la } \\
\text { información, de acuerdo a los } \\
\text { requerimientos, politicas, seguridad } \\
\text { de la organización. }\end{array}$ \\
\hline $\begin{array}{l}\text { Perfil Profesional } \\
\text { Administrador de } \\
\text { Base de Datos }\end{array}$ & $\begin{array}{l}\text { Modela, construye y gestiona la } \\
\text { arquitectura de datos de la } \\
\text { organización, utilizando programas } \\
\text { de administración de base de datos } \\
\text { que permitan administrar la } \\
\text { información, de acuerdo a los } \\
\text { requerimientos, politicas, } \\
\text { seguridaddela organización. }\end{array}$ \\
\hline Perfil Profesional & $\begin{array}{l}\text { Diseña, instala y gestiona redes } \\
\text { i n f o r m á t i a s y d e } \\
\text { telecomunicaciones, aplicando } \\
\text { conceptos y técnicas de } \\
\text { comunicación de datos y redes de } \\
\text { ordenadores, en condiciones de } \\
\text { calidad y seguridad, de acuerdo a la } \\
\text { realidad de la organización y las } \\
\text { regulaciones del entorno. }\end{array}$ \\
\hline $\begin{array}{l}\text { Administrador de } \\
\text { Redes, } \\
\text { Telecomunicaciones } \\
\text { e Infraestructura } \\
\text { de TI }\end{array}$ & $\begin{array}{l}\text { Comprende y aplica los principios } \\
\text { de las matemáticas y la ciencia a los } \\
\text { problemas de informática. }\end{array}$ \\
\hline $\begin{array}{l}\text { Aplicación de } \\
\text { matemáticas y } \\
\text { ciencias. }\end{array}$ & $\begin{array}{l}\text { Trabaja en equipo, interactuando } \\
\text { con los usuarios que intervienen en } \\
\text { el desarrollo de la solución } \\
\text { informática y participando de } \\
\text { manera efectiva en proyectos } \\
\text { informáticos. Comprende y aplica } \\
\text { los principios de las matemáticas y } \\
\text { la ciencia a los problemas de } \\
\text { informática. }\end{array}$ \\
\hline Trabajo en equipo & $\begin{array}{l}\text { Comunica de manera efectiva, de } \\
\text { forma oral, escrita y gráfica al } \\
\text { interactuar con diferentes tipos de } \\
\text { audiencia. }\end{array}$ \\
\hline Comunicación & $\begin{array}{l}\text { Comprende el impacto de las } \\
\text { soluciones informáticas en un } \\
\text { contexto globaly social, comprende } \\
\text { temas y responsabilidades } \\
\text { profesionales, éticas ylegales. }\end{array}$ \\
\hline $\begin{array}{l}\text { Responsabilidad } \\
\text { ética y profesional }\end{array}$ & $\begin{array}{l}\text { Se actualiza en los avances } \\
\text { tecnológicos de la profesión y se } \\
\text { involucra en un aprendizaje } \\
\text { continuo. }\end{array}$ \\
\hline
\end{tabular}

Se debe relacionar cada uno de los Cursos del Plan de Estudios con las Competencias Generales.

\section{Rf 5.2 Competencias Terminales de los Cursos}

Ejemplo de Competencias Terminales de los Cursos

\section{CURSO: FUNDAMENTOS DE SISTEMAS DE INFORMACIÓN COMPETENCIATERMINAL}

Diseña e implementa interfaces de usuario y reportes utilizando fundamentos de los sistemas de información y los aspectos técnicos involucrados en la construcción de los sistemas de información, valora la importancia que tienen los SI hoy en las empresas públicas y privadas.

\section{Rf 6: Matriz Curricular}

Se alinean las competencias con los Objetivos Educacionales Profesionales.

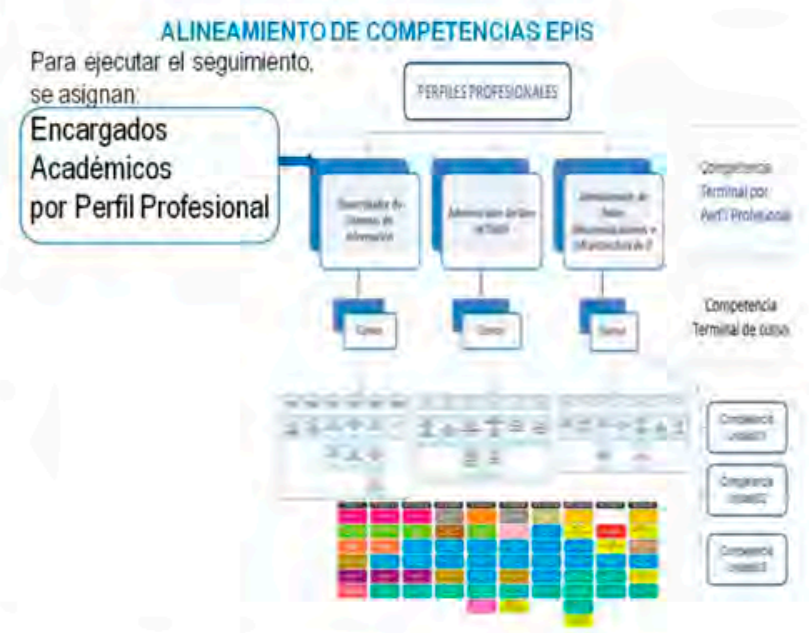

\section{Rf 7: Cursos y Otras Actividades de Aprendizaje}

El formato del silabo es para uso obligatorio en todos los cursos del Plan de Estudios de la Escuela Profesional de Ingeniería de Sistemas.

\section{PROCEDIMIENTO}

Aprobado por el Consejo de Facultad de Ingenieria

\section{Rf: 8 Ejecución de la Evaluación del Logro de las Competencias}

Para validar si se cumplió con el logro de las competencias y si se alcanzó las competencias definidas, es importante la opinión de los egresados. 
En la evaluación se busca determinar el impacto que puede tener el egresado de una carrera, con respecto a lo determinado en los perfiles profesionales propuestos; $\mathrm{y}$ a si soluciona problemas y satisface las necesidades que el ámbito socialle demanda.

La información que ofrecen los indicadores especifica con mayor precisión lo que se pretende evaluar.

Los indicadores se erigen en señales e indicios que apuntan hacia determinado logro.

\section{ACTIVIDAD 4:}

VALIDACIÓN DE REQUERIMIENTOS:

\section{Actividad 4.1: Técnicas de validación de requerimientos \\ Revisiones de requerimientos. \\ Los requerimientos son analizados sistemáticamente por un equipo de revisores, en este caso la Comisión Académica de la Escuela de Ingeniería de Sistemas revisa cada año los requerimientos.}

\section{Construcción de prototipos}

Es un enfoque de validación, muestra un modelo del sistema a los usuarios ya que pueden experimentar con este modelo para ver si cumple sus necesidades reales.

\section{Actividad 4.2: Gestión de requerimientos \\ Requerimientos duraderos y volátiles}

Desde una perspectiva evolutiva, los requerimientos se dividen en dos clases:

\section{- Requerimientos duraderos:}

Son requerimientos que se derivan de la actividad principal del Sistema y que están relacionados directamente con el dominio del sistema.

\section{- Requerimientos volátiles:}

Son requerimientos que probablemente cambian durante el proceso de desarrollo del sistema o después de que éste se haya puesto en funcionamiento.

Rf 9. Procedimiento de cambio: gestión de requerimientos

Identificar las brechas (los resultados del aprendizaje que no cumplen con los niveles de logro esperados (paso $8 \mathrm{del}$ modelo).

Realiza cambios para mejorar sus procesos educativos y eliminar las brechas (Paso 10).

Evalúa de nuevo para ver si los cambios fueron suficientes para eliminar las brechas, aplica encuestas a egresados (Paso 11).
Los pasos 10 y 11 se refieren a como "cerrar el ciclo" y la carrera tiene que hacer esto cada año.

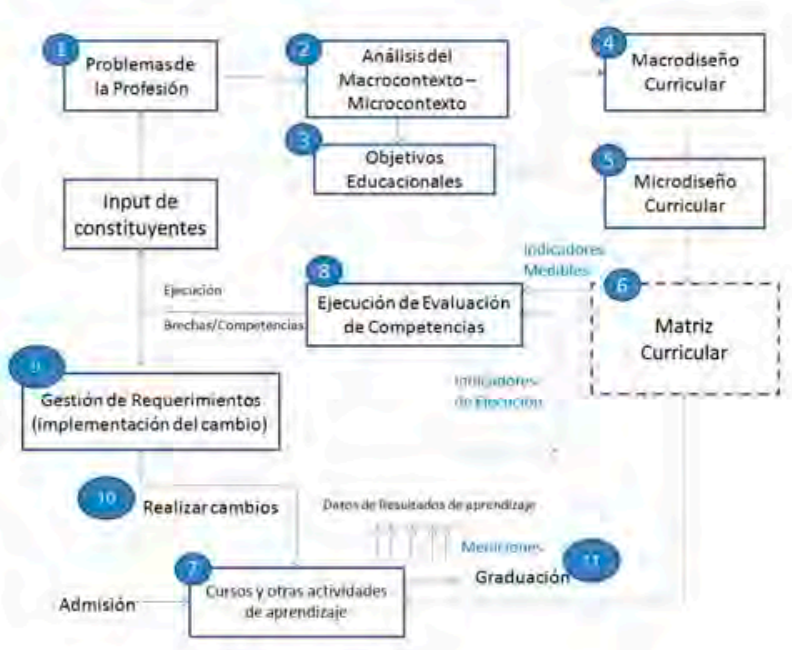

\section{RESULTADOS}

PRESENTACIÓN DE RESULTADOS DEL PROCESAMIENTO DE DATOS

En materia de evaluación de competencias los indicadores pueden orientarse hacia el dominio: conceptual y desempeño (la evaluación es una acción hacia la calidad).

La competencia se manifiesta a través de la actuación.

El logro, dado su carácter ideal, se manifiesta a través de indicadores que representan los aspectos específicos que van a ser evaluados.

Los indicadores miden los niveles de logro alcanzados.

Para validar si se lograron las competencias, es importante la opinión de los egresados, por lo que en cada ciclo de cambio se debe realizar el Análisis de los egresados y sus competencias.

Se han aplicado encuestas a los egresados, para determinar el logro alcanzado por las competencias y la proyección de eficacia de cada una de las competencias para los siguientes años.

Se han procesado las encuestas correspondientes a dos ciclos de cambios 2008 y 2009; y se ha proyectado la eficiencia de los egresados en las competencias para los años 2011 y 2012. 
INDICADORES DE LOGROS DE COMPETENCIAS

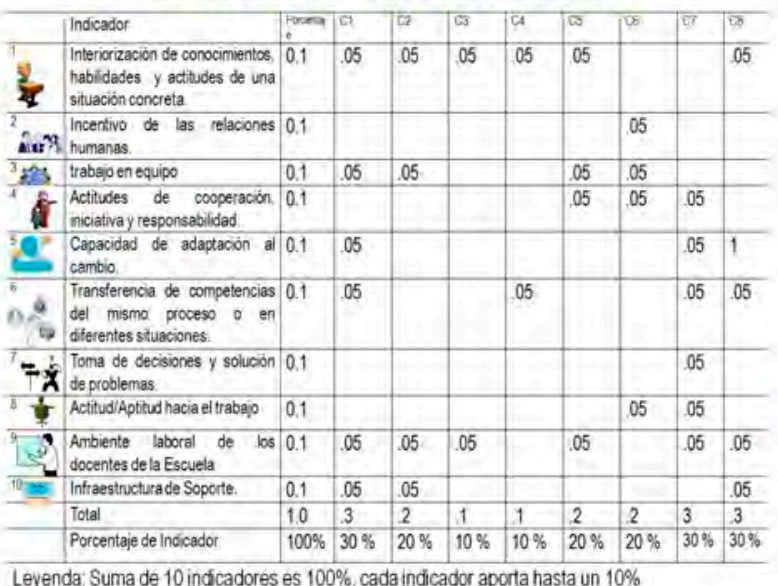

PROYECCIÓN DE LA EFICACIA DE LA COMPETENCIA:

APLICANDO EL INDICADOR POR COMPETENCIA $\mathrm{EN} \%$

\begin{tabular}{|c|l|c|}
\hline $\mathbf{N}$ & \multicolumn{1}{|c|}{$\begin{array}{c}\text { Competencia relacionada } \\
\text { a: }\end{array}$} & $\begin{array}{c}\text { Indicador } \\
\%\end{array}$ \\
\hline 1 & $\begin{array}{l}\text { Perfil Profesional } \\
\text { Desarrollador de Sistemas de } \\
\text { Información } \\
\text { Perfil Profesional } \\
\text { Administrador de Base de } \\
\text { Datos }\end{array}$ & 30 \\
\hline 3 & $\begin{array}{l}\text { Perfil Profesional } \\
\text { Administrador de Redes, } \\
\text { Telecomunicaciones e } \\
\text { Infraestructura de TI }\end{array}$ & 10 \\
\hline 4 & $\begin{array}{l}\text { Aplicación de matemáticas y } \\
\text { ciencias. }\end{array}$ & 10 \\
\hline 5 & \begin{tabular}{l} 
Trabajo en equipo \\
\hline 6
\end{tabular} & Comunicación \\
\hline 7 & $\begin{array}{l}\text { Responsabilidad ética y } \\
\text { profesional }\end{array}$ & 30 \\
\hline 8 & Aprendizaje continuo & 30 \\
\hline
\end{tabular}

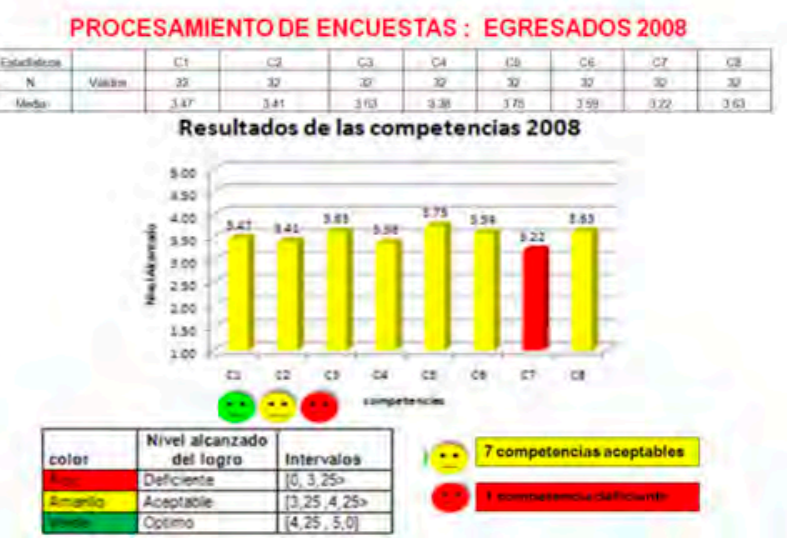

Tomando como base, los resultados del año 2008, se proyecta un incremento de logro por competencia para el año 2011.

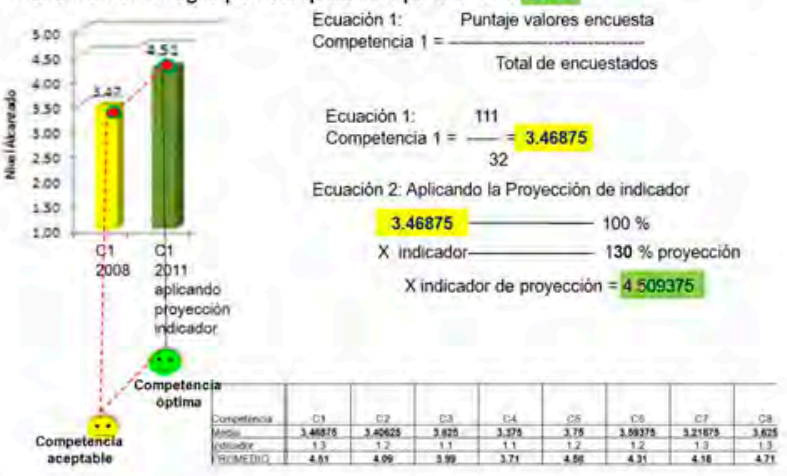

Proyección de Competencias 2011

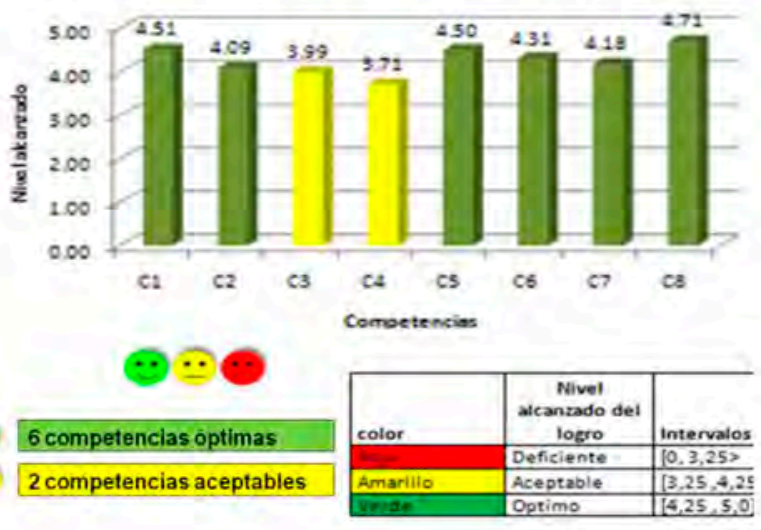

La aplicación de la Ingeniería de requerimientos, ha permitido establecer mecanismos y control de cambios de las 8 competencias generales de Ingeniería de Sistemas; lo que permitió establecer una proyección por cada una de las competencias, determinando que 6 competencias alcanzarán un logro óptimo y 2 competencias alcanzarán un logro aceptable. 


\section{DISCUSIÓN}

Con respecto a la Sub Hipótesis "A" que dice que se encuentran carencias en los recursos metodológicos del Diseño Curricular de la EPIS de Ia UPT, en los aspectos que no se implementó un Perfeccionamiento Curricular aplicando la Ingeniería de Requerimientos, por lo que se consideró los requerimientos dinámicos del entorno.

En el cuadro "Actividades Ocupacionales del Ingeniero de Sistemas" se aprecian las tendencias a las actividades relacionadas al Desarrollo de Sistemas de Información.

Se han identificado los requerimientos dinámicos, lo cual se ve reflejado en al Cuadro Clasificación de los requerimientos volátiles. Asimismo, se han determinado los requerimientos Cambiantes y Emergentes, los cuales son evaluados cada ciclo de revisión por los integrantes de la Comisión Académica, lo que permitirá contar con un Plan de Estudios actualizado. Esta obtención de los requerimientos dinámicos del entorno ha permitido establecer los Perfiles Profesionales para la demanda del contexto.

Los porcentajes de las áreas de conocimiento, gracias a la captura de requerimientos, en el Plan de Estudios resultado de esta investigación son los siguientes:

- Núcleo de Sistemas de Información (21\%)

- Ambiente de Sistemas de Información (15\%)

- Sistemas de Información Avanzado (31\%)

Con respecto a la Sub Hipótesis " $\mathrm{C}$ " relacionada al no haber incorporado al currículo los requerimientos dinámicos, y establecer responsables de la medición de los indicadores del logro de las competencias terminales EPIS UPT, es causa de las distorsiones entre el logro alcanzado y el logro proyectado por los egresados de la Escuela de Ingenieria de Sistemas de la Universidad Privada de Tacna.

Para validar si su cumplió con el logro de las competencias, es importante la opinión de los egresados; por lo que en cada ciclo de cambio se debe realizar el Análisis de los egresados y sus competencias. Se han aplicado encuestas a los egresados para determinar el grado alcanzado por las competencias y la proyección de eficacia.

\section{Se han procesado encuestas a dos ciclos de cambios:}

\section{Del 2008:}

Según la semaforización: en amarillo Nivel Aceptable hay 7 competencias; y 1 competencia en rojo, Nivel Deficiente.
Aplicando la proyección, según la semaforización en verde Óptimo se proyectaron 6 competencias; y en amarillo Aceptable, 2 competencias para los egresados para el 2011.

\section{Del 2009:}

Según la semaforización en amarillo Nivel Aceptable hay 6 competencias; y 2 competencias en rojo, Nivel Deficiente,

Aplicando la proyección, según la semaforización en verde Óptimo se proyectaron 6 competencias; y en amarillo Aceptable, 2 competencias para los egresados para el 2012.

Los cuales contribuyen a satisfacer la demanda actual de actividades ocupacionales, que según el cuadro 6 Tendencias Ocupacionales están orientadas al Desarrollo de Sistemas de Información, donde la demanda de actividades ocupacionales guarda relación con los cursos ofertados en el Plan de Estudios resultado de esta investigación.

Con respecto a la Sub Hipótesis "D" que dice que se encuentran deficiencias en las actividades de ejecución de las competencias terminales EPIS debido a que no se utilizaron indicadores para conocer el nivel del logro de las competencias; las apreciaciones resultantes son:

En la figura formato de encuesta egresados:

Se procesaron las encuestas para conocer el nivel alcanzado de las competencias, por los egresados.

Para validar si se lograron las competencias, es importante la opinión de los egresados, por lo que en cada ciclo de cambio se debe realizar encuestas de sus competencias.

\section{En Ia FIGURA ALINEAMIENTO DE COMPETENCIAS EPIS:}

Las competencias terminales de la carrera y las competencias de los cursos, se alinean y al aplicar el Control de Cambios en Requerimiento Funcional 6: Revisión de la Matriz Curricular, en la Matriz de Evaluación de Competencias, se evalúan las competencias.

La propuesta de nuevas lineas de investigación se enmarcan dentro de las condiciones del contexto mundial y el entorno que afecta a la formación del Ingeniero de Sistemas, que debe orientarse a la formación para Ingenieros que cumplan requerimientos globales; por tanto, lineas desarrolladas para articular diseños curriculares contextualizados a requerimientos cambiantes y globales. 
Los responsables de Escuelas de Ingeniería de Sistemas deben tener una visión clara de los productos, es decir, de los egresados que se desea obtener, más una buena medida de convicción y perseverancia.

Los responsables de implementar el Perfeccionamiento Curricular deben hacer un seguimiento y monitorizar los procedimientos y mecanismos establecidos por la Ingeniería de Requerimientos; incorporar sistemática y metodológicamente las caracteristicas que debe reunir el futuro Ingeniero de Sistemas Global, competente en materias técnicas, servidor de la comunidad mundial, buen comunicador, que trabaje en equipo y esté preparado con las capacidades más complejas e integradas requeridas.

\section{REFERENCIAS}

1. Ingeniería de Software Ian Somerville Séptima edición Editorial Pearson Educación S.A. Madrid 2005. Capitulo 8 página 154.

2. DISEÑO CURRICULAR Metodología para el perfeccionamiento del currículum en su esfera de acción DRA. GLADYS CARIDAD TORRES ESTÉVEZ. 